\title{
Salud, trabajo y ambiente. Estrategias para el desarrollo regional
}

\author{
JORGE PICKENHAYN \\ Universidad Nacional de San Juan, Argentina. \\ picken@infovia.com.ar
}

\section{RESUMEN}

Toda acción humana genera un impacto en el ambiente. Los bienes de la naturaleza se re-significan cuando la sociedad les asigna el nombre de «recursos». También los modelos productivos implican «recurrir» a estrategias concretas que traen consigo necesarias acciones que producen cambios. Hay permanentes umbrales que establecen el quiebre entre un antes y un después para cada paisaje.

La sociedad tiene metas que pone en juego para determinar sus proyectos de desarrollo, particularmente si las mejoras perseguidas involucran al territorio. Cuando nos preguntamos cuáles son esas metas aparece el «mito del Edén»: la respuesta es «vivir», concepto demasiado amplio y complejo que puede simplificarse si lo dividimos en: vivir bien, sano y mucho. Todo modelo productivo se inspira en el trabajo como instancia de transformación (o sea, vivir mejor). Para ello su agente, el hombre, necesita preservarse y perdurar (es decir, vivir sano y mucho).

Cuando los especialistas en planeamiento piensan modelos de ordenación territorial para grandes superficies realizan comparaciones de muy diversa factura y concluyen en modelos de síntesis para el presente y sobre el futuro planificado. Se suelen, empero, descuidar los vínculos entre salud, trabajo y ambiente. Estimamos que esta triangulación es importante por lo que se propone una metodología de planificación que tenga en cuenta: a) el estudio de los impactos recíprocos entre salud, ambiente y trabajo; b) el análisis de problemas concretos asociados con la distancia y accesibilidad a lugares de empleo y centros de salud en relación con los espacios de residencia de la población, y c) propiciar un ordenamiento espacial que potencie los vínculos y minimice las dificultades por la relación de estos tres factores. Los puntos destacados merecen tratarse con mayor peso en las estrategias de desarrollo regional que propicien un planeamiento que apunte al desarrollo productivo.

PALABRAS CLAVE: salud; trabajo; desarrollo; ambiente. 


\section{ABSTRACT}

\section{HEALTH, WORK AND ENVIROMENT. REGIONAL DEVELOPMENT STRATEGIES}

Every human action has an impact on the environment. The society resignifies the goods the nature provides by calling them «resources». Production models also involve taking action that brings necessary changes. There are permanent thresholds that show the difference in time of each landscape.

The society has objectives to establish its development projects, and it is particularly interested in improvements that involve the territory. When we wonder which those objectives are, the «myth of Eden» appears: the answer is «to live», a concept too broad and complex that can be simplified if we divide it into: live well, healthy and long. Every production model is inspired by work as an instance of transformation (i.e. live better). In order to accomplish this, the man needs to endure and preserve himself (i.e. live healthy and long).

When planning specialists think about spatial models for large areas, they make many comparisons. They conclude synthesis models for the present and the future planned; but they tend to neglect the links between health, labour and environment. We estimate that this triangulation is important, so a planning methodology is proposed to take into account: a) the study of the reciprocal impacts between health, environment and labour; $b$ ) the analyses of specific problems associated with distance and accessibility to workplaces and health centres in relation to the spaces of residence of the population, and c) the promotion of a spatial planning to reinforce the links and to minimize the difficulties arisen due to these three factors. The highlights deserve to be treated in depth within regional development strategies that foster a planning point for a productive development.

KEYWORDS: health; work; development; enviroment. 


\section{RESUMO}

\section{SAÚDE, TRABALHO E AMBIENTE. ESTRATÉGIAS DE DESENVOLVIMENTO REGIONAL}

Toda ação humana gera um impacto no ambiente. Os bens da natureza obtêm novo significado quando a sociedade lhes dá o nome de «recursos». Também os modelos produtivos implicam «recorrer» a estratégias concretas que trazem ações necessárias, as que produzem mudanças. Há permanentes umbrais que estabelecem o quebre entre um antes e um depois para cada paisagem.

A sociedade tem metas que põe em jogo para estabelecer seus projetos de desenvolvimento, particularmente se as melhoras perseguidas envolvem ao território. Quando nos perguntamos quais são aquelas metas aparece o «mito do Éden»: a resposta é «viver», conceito muito amplo e complexo que pode se simplificar, se o dividimos em: viver bem, são e muito. Todo modelo produtivo inspira se no trabalho como instancia de transformação (o seja, viver melhor). Para isso seu agente, o homem, precisa se preservar e perdurar (o seja, viver são e muito).

Quando os especialistas em planejamento pensam modelos de ordenação territorial para grandes superfícies realizam comparações de muito diversa fatura e concluem em modelos de síntese para o presente e sobre o futuro planejado. Mas às vezes se descuidam os vínculos entre saúde, trabalho e ambiente. Estimamos que esta triangulação é importante e propõe se uma metodologia de planificação que tomem em conta os pontos a seguir: a) o estudo dos impactos recíprocos entre saúde, ambiente e trabalho; b) o análise de problemas concretos associados à distancia e acessibilidade a lugares de trabalho e centros de saúde com relação aos espaços de residência da população, e c) propiciar um ordenamento espacial que potencie os vínculos e minimize as dificuldades pela relação entre estes três fatores. Os pontos destacados merecem ser tratados com maior peso nas estratégias de desenvolvimento regional que propiciem um planejamento orientado ao desenvolvimento produtivo.

PALAVRAS-CHAVE: saúde; trabalho; desenvolvimento; ambiente. 


\section{Una mirada sobre el desarrollo}

Es habitual que las iniciativas para mejorar las condiciones de desarrollo en un país o una región movilicen ideas destinadas a sustentar una plataforma teórica sobre la que luego se habrá de construir. En estos marcos, ciencia y política convergen para buscar puntos de partida. El primero radica en el quid de la cuestión y se desdobla en algunas preguntas básicas: ¿qué es desarrollo regional? ¿Dónde nos proponemos actuar? ¿Cómo lo haremos? ¿Por qué allí?

Existe desarrollo en tanto hay energías en potencia que prometen que algo se despliegue favorablemente (por eso la palabra portuguesa desenvolvimento es más sugerente que la nuestra). El desarrollo es transformación y perfeccionamiento pero además supone una acción determinada, algo así como un disparador que pone en funcionamiento un conjunto de fuerzas que estaban implícitas antes y que de este modo se organizan para formar un sistema capaz de procesar energía y darle impulso.

El desarrollo regional es un tema medular para la geografía aplicada en tanto propone formas originales de ordenación del territorio. La noción teórica de región, que para los geógrafos se basa en aquellos espacios que tienen una presencia territorial con definida cohesión y límites concretos, también tiene una vertiente asociada con el regionalismo. Allí, como dirá Pasquier, se conjugan la idea de «colectividad territorial» con la de «espacio económico» (1970:44-45). Es en esta última forma de ver las cosas que se despliegan las competencias institucionales, la participación social y la acción administrativa para modificar el ambiente. Esto, que la geografía postula desde hace medio siglo, es lo que debería ser el desarrollo regional.

Cuando este asunto comenzaba a debatirse entre los especialistas, ya se marcaba la necesidad de diferenciar entre los cambios de larga y corta duración (Braudel, 1958). Para desarrollar una región es necesario llevar a cabo una planificación sectorial, consistente en la extrapolación de datos del pasado cercano para corregir, con mínimos golpes de timón, la marcha hacia el futuro (también cercano). Esta acción —una suerte de ingeniería social fragmentaria, como la denominara Popper (1984:79) — se complementa con otra, de proyecto más largo, donde los efectos no sólo se pueden ver con el correr del tiempo; además se van potenciando desde el momento inicial, operado ex nibilo, desde cero (Randle, 1978:69). La fórmula aplicada por Jean Labasse era muy sugerente: antes que nada hay que «comprender la duración y las proporciones» (I973). 
A más de medio siglo del comienzo de las prácticas profesionales de planeamiento territorial, las acciones desarrolladas a nivel estatal dieron como resultado muchos modelos de aplicación, organizados mediante rutinas de intervención. Así se definen metas que apuntan a cambiar el territorio. La respuesta buscada coincide con la panacea clásica del Edén: en última instancia lo que se persigue es que la gente viva mejor (resumida en esa palabra de poco asidero científico y mucha carga emotiva que es la felicidad).

Se trata, obviamente, de una utopía que puede descomponerse en varias facetas, donde intervienen necesidades humanas. Vivir bien implica vivir en libertad, vivir en compañía, con seguridad, con equidad, con comodidad, progresar, relacionarse, acceder a la naturaleza y la cultura, entretenerse y tantas otras aspiraciones que sería extenso enumerar. En lo más profundo de esta desiderata se visualizan dos aspectos que comentaremos con más detenimiento: vivir mucho y vivir sano. Es un dicho popular que la salud se valora cuando se pierde. A esto hay que agregarle el peso simbólico del mito de la inmortalidad: no somos conscientes de la finitud a pesar que sabemos que la muerte es inexorable. El hombre, decía Sartre (1946), es el único espécimen de la naturaleza que sabe que va a morir... Pero, agregamos nosotros, siempre hace planes para la vida, nunca para la muerte.

Vivir sano y mucho marca la meta de las ciencias de la salud, gran objetivo que en algunos aspectos se cruza con el ambiente, que ya no es el cómo sino el dónde se vive. Vivir aquí o allá es, ya en un principio, tomar una decisión geográficamente relevante. Además lo es el acto de cambiar el espacio o adaptarse a él, ya sea para mejorar las condiciones de habitabilidad, usar los recursos (y consecuentemente protegerlos), favorecer las relaciones sociales en el territorio o prever mejores formas de adecuación para el futuro.

Desde la simple apropiación de alimentos hasta las formas más sofisticadas de acumulación, el hombre recurre al trabajo como alternativa productiva. Esta actividad humana —originalmente una dinámica necesaria para acceder a los bienes, transformarlos y ponerlos a disposición del sistema - se descompone en dos grandes categorías: una productiva (donde aparecen las actividades extractivas, agropecuarias e industriales) y otra relativamente estéril (una amplia gama de servicios donde predomina el comercio). 
El trabajo, al igual que la salud, está condicionado por estructuras ambientales, puesto que toda actividad humana, por nimia que parezca, depende de la localización de los recursos, de los operarios, de los potenciales destinatarios y, en definitiva, de la empresa que organiza este sistema, como dicen las viejas reglas de Alfred Weber (1922).

Con el desarrollo regional, las innovaciones que producen cambio y adelantos, también son responsables de una verdadera revolución geográfica, en la medida en que favorecen traslados y nuevas valorizaciones del espacio. Las condiciones naturales que constituían la base material de la existencia del grupo cambian con el impulso recibido y aparecen nuevas exigencias ambientales. Como señala Soares de Olivera, estos cambios a menudo están relacionados con el crecimiento urbano: "Las innovaciones técnicas y tecnológicas excluyeron y excluyen a aquellos que no tienen la formación necesaria para el ejercicio de las nuevas funciones, forzándolos a migrar hacia otros lugares, por lo general áreas urbanas» (2007:86-87).

Los argumentos hasta aquí presentados nos permiten fundamentar la fuerte conexión entre los elementos de un sistema que debería estudiarse integralmente, donde se asocian ambiente, salud y trabajo, en un contexto de desarrollo. Es necesario aquí establecer una diferenciación importante: no se trata de tres categorías homogéneas. El ambiente es un contexto; el trabajo, una actividad, y la salud es un bien.

\section{Falencias de la planificación en materia de salud y trabajo}

La planificación territorial ocupó a los argentinos en muchas etapas de la historia reciente. Hay interesantes aportes en investigaciones oficiales y particulares, algunos de ellos transformados en cuadernos, mapas, libros y hasta leyes nacionales y provinciales. Lamentablemente, muy pocos de estos esfuerzos se volcaron en acciones concretas, a veces por falta de continuidad política, otras por desacuerdos institucionales y finalmente, muy a menudo, por la necesidad de realizar emprendimientos urgentes que obstruyeron los planes de largo y mediano plazo, de fundamental importancia estructural.

Entre estas iniciativas pueden contarse numerosas variantes, las primeras de las cuales fueron sistematizadas por Rey Balmaceda (1972:106 et passim) bajo la denominación de «espacios polarizados», clasificados 
como "regiones plan y programa» en alusión a la intervención estatal y privada, respectivamente. De esas experiencias iniciales se destaca el Sistema Nacional de Planeamiento y Acción para el Desarrollo creado por la Ley 16964 de 1966, que permitió generar ocho «regiones de desarrollo» establecidas por Decreto I097/67 y que tuvieron trascendencia posterior con el nombre popular de regiones del CONADE (porque se definieron como instrumento de planeamiento por el Consejo Nacional de Desarrollo).

En estos casos, la geografía voluntaria ejerce una intervención inspirada en la soberanía territorial «a fin de llevar a cabo decisiones políticas conducentes a una mejor utilización del espacio» (Roccatagliata, 1982:35).

En los gobiernos democráticos recientes hubo iniciativas de planeamiento territorial, aunque en la mayoría de los casos tuvieron el liderazgo de arquitectos y no de geógrafos. Estas planificaciones, concebidas con un criterio integral, tienen un rasgo común. Salvo detalles excepcionales, todas ellas tienen falencias en los campos de la salud y el trabajo.

\section{El Plan Estratégico actual}

Analizaremos en particular el proyecto vigente en el orden nacional denominado PET, abreviatura de «Plan Estratégico Territorial» (Argentina, 2008, 20IO, 20II y Kossoy, 20IO). Se lo definió institucionalmente como: «Proceso de planificación estratégica conducido por el gobierno nacional, mediante la formación de consensos, para el despliegue de la inversión pública y el desarrollo territorial» y lo lleva adelante un equipo técnico de la Subsecretaría de Planificación Territorial de la Inversión Pública del Ministerio de Planificación Federal, Inversión Pública y Servicios dependiente del Poder Ejecutivo Nacional. 


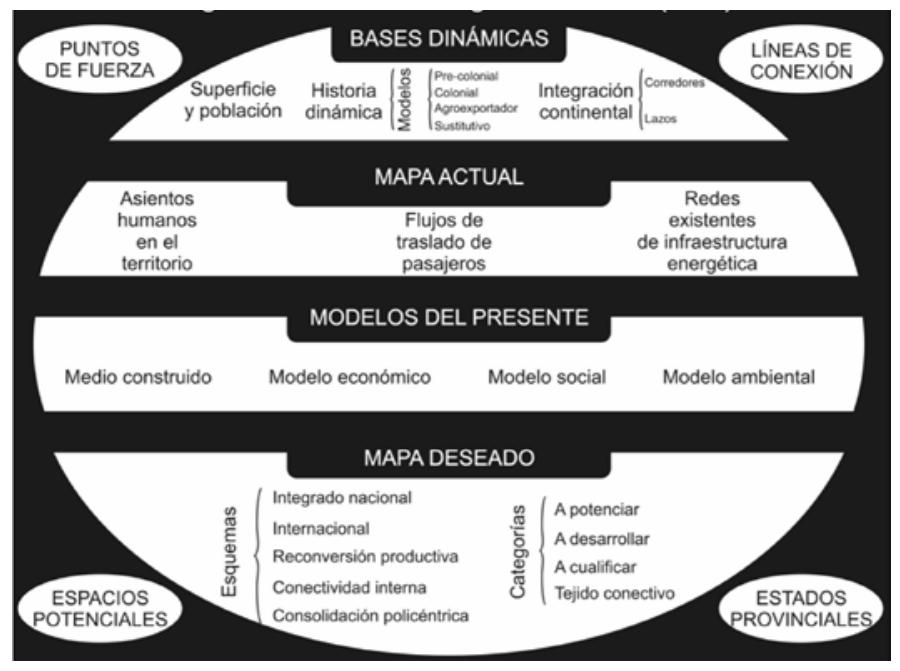

Fuente: Elaboración propia

Figura 1. Argentina. Plan Estratégico Territorial (PET)

Su estrategia se organiza más en torno a puntos de fuerza, líneas de conexión y espacios potenciales — conceptos propios del diseño-que a regiones plan o espacios polarizados. Tal vez esto sea así porque, como queda dicho, en el equipo predominan profesionales no geógrafos.

Los efectos, sin embargo, son interesantes, ya que es auspicioso, como se señala en la profusa documentación aportada por el plan, que los resultados del mismo se utilicen para «identificar las inversiones en infraestructura y equipamiento necesarias para el desarrollo territorial de la Nación y las Provincias» (Kossoy, 20IO:I). En una palabra, no se trata de un anhelo utópico diseñado en vistosas cartografías sino la base para establecer prioridades políticas en la ejecución de obras de infraestructura.

El planeamiento se basa en la comparación inicial de un modelo que refleja la actualidad y otro "deseado", construidos ambos a partir de factores y procesos intervinientes en el territorio actual y proyectados en ideas rectoras y líneas de acción integradoras (PET, 2008, capítulos 2 y 3 , respectivamente).

Existe un enfoque general que contempla al país en su conjunto y etapas para las provincias generadas por acuerdo con cada Estado. La perspectiva presentada en el primer avance publicado en 2008 se ajusta en el segundo, producido un lustro después (PET, 20I3). 
Las características del PET pueden sintetizarse del siguiente modo: se definen primero las jurisdicciones provinciales con sus datos elementales de superficie, población y densidad; se realiza luego un estudio de la evolución histórica del territorio - modelos precolonial, colonial, de principio de siglo XX (en la versión actualizada se lo reemplaza por el modelo agroexportador) y de la etapa de sustitución de importaciones-. Se muestran luego los corredores de integración continental y los lazos internacionales, para llegar al denominado «mapa actual», que refleja la dinámica potencial para una ordenación del territorio. Continúa el estudio de los asientos humanos en el territorio, sus flujos de traslado de pasajeros y redes de infraestructura energética, que definen el mapa sobre «medio construido».

A partir del concepto de infraestructura del territorio se comparan densidades por departamento con infraestructura vial, ferroviaria, puertos, frecuencia de vuelos comerciales y aspectos complementarios como pasos internacionales y red telefónica.

En una etapa posterior se presenta un "modelo económico» que se apoya en mapas como la tasa de desempleo o la participación provincial en el producto bruto geográfico. En la cartografía de 2013 aparece un mapa interesante que define dinámicas económicas con áreas industriales y agropecuarias con distintos niveles de posibilidad de industrialización, pero no se reflejan allí las situaciones generadas por la minería, especialmente del petróleo, el oro, el hierro y el carbón, donde colisionan aspectos laborales, de la salud y el ambiente.

Le sigue el «modelo social», que, como el económico, define espacios de tres categorías, en relación con las condiciones favorables, intermedias y desfavorables. Acá se exponen cartas que reflejan tasas de dependencia demográfica, déficit de cobertura de agua de red, de cloacas y hacinamiento en los hogares, población con cobertura de obra social, nivel de instrucción alcanzado y distribución de la población urbana y rural.

$\mathrm{El}$ «modelo ambiental» define niveles altos, medios y bajos de vulnerabilidad y su inserción en las eco-regiones del país. Se sustenta esta etapa con cartas de potencialidades y restricciones ambientales, comparadas a su vez con el uso de la tierra, los sistemas de cuencas hidrográficas y la superficie afectada por incendios.

$\mathrm{El}$ «modelo deseado» que aparece como visión de síntesis y base de planificación, se inspira en cinco esquemas simples en los que se expresan las ideas rectoras para el ordenamiento: a) el integrado nacional; b) el internacional; c) el de reconversión productiva; d) el de conectividad 
interna, y e) el de consolidación de ciudades con criterio policéntrico. El resultado "deseado» es una malla de espacios divididos en dos categorías: aquellos que deberían potenciarse y los que necesitan desarrollarse (se incluyen también los que aún están por cualificarse), todos ellos insertos en lo que se denomina "tejido conectivo", que en algunos casos, como Buenos Aires y Santa Fe, casi no existe, y en otros, como San Juan, Formosa y Chaco, representa más del $50 \%$ de su superficie.

En los trayectos provinciales se repite la falencia de aspectos referidos a la salud. Algunos Estados, como Buenos Aires, se limitan a incluir las áreas de salud (viejas divisiones que están en etapa de reforma) y el número de centros por partido, dato poco significativo si no se lo asocia con la densidad y la accesibilidad. En el ejemplo comentado, hay mapas de desocupación y de tipos de actividad económica, pero estos datos tienen poca incidencia en las cartas prospectivas. Tanto el trabajo como la salud son poco considerados.

El esquema provincial de ordenamiento territorial propuesto para San Juan (en el cual sí interviene una geógrafa, la doctora mendocina Nelly Gray de Cerdán, quien desarrolló el informe final, según aparece en la publicación) define cinco líneas de crecimiento donde no se observa una incidencia directa de los temas de salud y trabajo. El fundamento reside en la búsqueda de una suerte de equilibrio sustentable entre fortalezas y debilidades respecto del desarrollo, en términos de conexión, organización y activación. Estas líneas son:

- Fortalecer y reorganizar la zona Norte, que incluye Iglesia y Jáchal.

- Equilibrar la zona Sur, que incluye los valles de Tulum, Ullum y Zonda.

- Renovar y reactivar la zona Oeste, en el área de Calingasta.

- Conectar y lograr la autosustentabilidad de la zona Este, en el Valle Fértil.

- Establecer dos nodos urbanos de primer nivel, Gran San Juan y San José de Jáchal, encargados de la coordinación de actividades.

Algo similar ocurre en la populosa provincia de Santa Fe: el capítulo sobre salud tomado como antecedente apenas refleja algunos índices y tasas obtenidos de otras fuentes, con escasa incidencia en los mapas.

El símbolo que alerta sobre dificultades de acceso a los centros, en los mapas integrados (una cruz, en la amplia lista de referencias) apenas aparece tres veces en las provincias comentadas. 
En síntesis, consideramos muy auspiciosa la existencia y ejecución continua del PET en Argentina, y más aún por cuanto constituye una base estratégica para definir la ejecución de obras concretas. Observamos, sin embargo, que podrían valorizarse más los aspectos referidos a la salud y al trabajo en la planificación.

\section{Cómo valorizar salud y trabajo}

A esta altura sería importante definir cuáles serían los rasgos a fortalecer, para lo que volvemos a retomar las ideas iniciales referidas a la aspiración humana de vivir sano, mucho (aquí aparece la salud) y bien (con necesidad de trabajar para obtener recursos, en los planos individual familiar y colectivo).

En cuanto a salud, existen varios aspectos que deben considerarse en una planificación para el desarrollo. Las escalas de representación deberían responder como mínimo a los niveles provincial y departamental. Los ordenamos con un criterio de importancia para visualizar mejor las alternativas de operación:

A) Distribución de la pobreza.

a) Mapa de Necesidades Básicas Insatisfechas, por departamento.

b) Localización de los espacios que están por debajo de la mediana en su relación pobres/habitantes.

B) Distribución de los servicios de salud.

a) Presencia.

i. Regionalización existente.

ii. Número de establecimientos según niveles de complejidad.

iii. Densidad (relación cama/habitante — denominada técnicamente rotación- enfermero/habitante y médico/habitante).

iv. Tiempo de espera promedio en servicios ambulatorios.

b) Accesibilidad a los servicios de salud.

i. Distancia media al centro más próximo.

ii. Ambulancias. Relación tiempo/distancia.

iii. Traslados de alta complejidad.

iv. Acceso a las comunicaciones en centros alejados. 

c) Obra social.
i. Porcentaje de cobertura.
ii. Relación con el campo laboral.

C) Mortalidad y morbilidad.

a) Causas de muerte.

b) Morbilidad.

c) Semanas epidemiológicas.

d) Años potenciales de vida perdidos.

D) Eficiencia del sistema.

a) Relación público/privado.

b) Equipamiento de alta complejidad.

c) Controles de bioingeniería.

d) Prioridades no resueltas.

iii. Edilicias.

iv. Tecnológicas.

v. Déficit de asistencia.

Los aspectos laborales que deberían tenerse en cuenta en una planificación son los siguientes:
a) Mapa del desempleo.
b) Planes y asignaciones sociales.
c) Ocupación según actividad.
i. Primaria.
ii. Secundaria.
iii. Terciaria.

iv. Comparación entre receptividad laboral de empresas y población.

d) Población con posibilidades de acceder a la medicina privada (doble decil de la población con mayores recursos)

e) Traslados.

i. De operarios urbanos.

ii. Por trabajo rural.

f) Invisibilidad laboral.

i. Trabajo informal.

ii. Trabajo esclavo. 
iii. Trabajo afectado por contaminantes.

iv. Relación entre ocupados y afectados a planes.

v. Relación entre trabajadores formales e informales.

Fuente: Elaboración propia

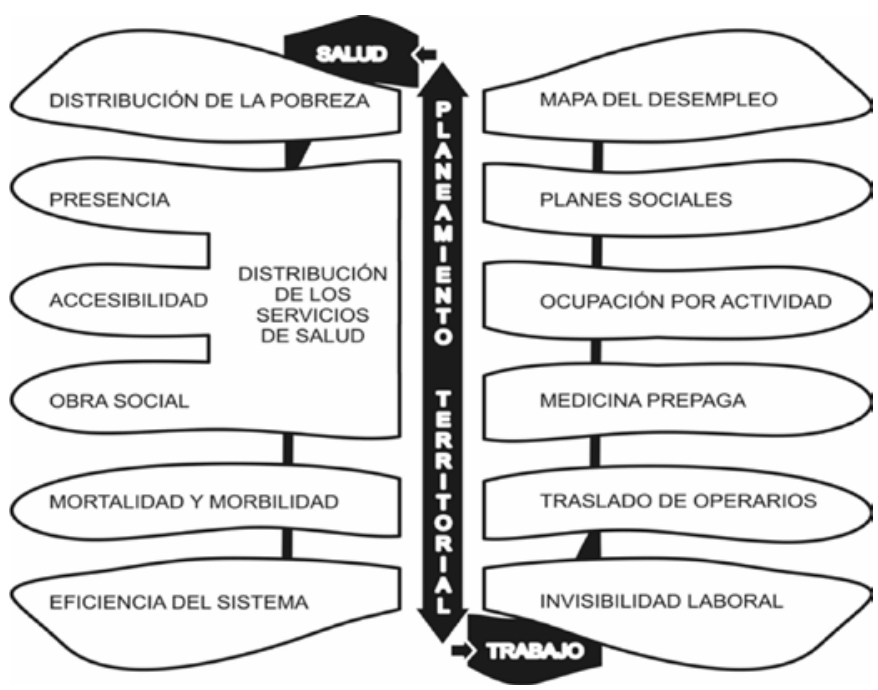

Figura 2. Valorización de la Salud y el Trabajo

\section{Rasgos comunes}

Hay, además, elementos comunes en ambas problemáticas que en unos casos pertenecen a campos diferentes y en otros a la relación entre ambos:

a) Comunicaciones.

b) Red vial.

c) Relación sueldo/costo de obras sociales.

d) Proporción de costos entre obras sociales y prepagas.

e) Contaminación en ámbitos laborales.

f) Eficiencia de los sistemas de salud promovidos por las empleadoras.

Es interesante observar la semejanza entre las vías de desarrollo con los corredores que vinculan a trabajadores con sus lugares de trabajo y los corredores de salud, responsables del traslado de vectores. Pueden utili- 
zarse los mismos patrones espaciales en los tres casos, aunque son diferentes las pautas de ordenamiento que deben aplicarse. Para simplificar este aserto, podría decirse que la acción planificadora responde a objetivos a veces contradictorios: los corredores de desarrollo, por lo general, "se abren»; los de circulación laboral «se mantienen expeditos», y los de salud «se interrumpen». Lo interesante radica en que, a menudo, estos corredores coinciden, a veces sólo en algunos de sus tramos componentes y otras en su totalidad.

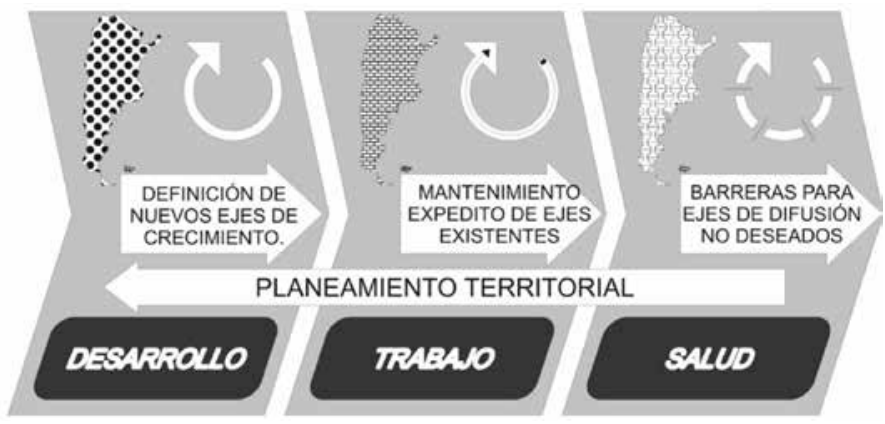

Fuente: Elaboración propia

Figura 3. Vínculo entre patrones espaciales

Proyectado un eje de desarrollo, cabe esperar que su presencia movilice circuitos de operarios que se trasladan por él, transportistas que conducen las mercaderías, circuitos de trabajadores movilizados y todo un sistema humano que genera contaminación, produce degradación del territorio y modifica las condiciones originarias del paisaje. Además, estas mismas personas llevan y traen enfermedades transmisibles y producen otros efectos concurrentes, como accidentes (principalmente de tránsito), deslocaciones transitorias o definitivas, déficit de servicios de salud en los nuevos sitios de concentración y sus problemas administrativos relacionados (cobertura social, acceso a medicamentos, etcétera).

En el caso del sistema nacional de salud, existe una tendencia descentralizadora que no debería alcanzar la instancia departamental para evitar duplicación de servicios e inversiones innecesarias de equipamiento complejo. Los informes del PET reconocen que «la trama compleja a la que ha dado lugar la descentralización del sistema en las jurisdicciones provinciales y municipales, dificulta su certera evaluación» (2008:72).

A estos problemas se suman los procesos naturales que generan corredores geoepidemiológicos y que suelen condicionar también el trazado 
de los ejes de desarrollo. Por ellos se movilizan los vectores y agentes de patologías que en su origen fueron predominantemente silvestres pero que en la actualidad responden a los procesos de organización que los mismos factores de desarrollo promueven en el espacio.

Es interesante observar la correspondencia que existe entre modelos de desarrollo con vías de comunicación y con corredores geoepidemiológicos. No sólo es interesante la coincidencia entre éstos: si se superponen las tres redes aparecen con claridad los espacios de conectividad, que se muestran como áreas muertas o de escasa circulación.

Los proyectos de desarrollo deben tener en cuenta no sólo la apertura de nuevos ejes sino la «limpieza» de aquellos existentes, por los que debe trasladarse la fuerza de trabajo y sus procesos colaterales y aun la interrupción con barreras de aquellos flujos de transmisión epidemiológica que circulan con simultaneidad a las pulsiones del crecimiento y que pueden afectarlo.

\section{Conclusiones}

De lo expuesto surgen algunas líneas de acción directa de fuerte incidencia territorial que un ordenamiento orientado hacia el desarrollo necesita. Se resumen así:

a) Caracterización ecológica y sanitaria del empleo potencial para promover el trabajo de bajo impacto y desalentar el que contamina o enferma.

b) Fortalecimiento en áreas que pueden mejorar su oferta laboral.

c) Optimización de posibilidades para movilizar mano de obra, equipos e infraestructuras empresariales.

d) Preservación de tradiciones laborales que generan especialidades.

e) Activación de fuentes de trabajo y su requerimiento en tecnología e infraestructura.

h) Perfeccionamiento del acceso al transporte y las comunicaciones.

i) Promoción de corredores que favorezcan los accesos más eficientes al trabajo y la salud.

j) Definición de barreras de control epidemiológico a través de la infraestructura que su implementación requiera.

En todos los casos se observa la necesidad de encaminar pequeñas pero eficientes operaciones de planificación sectorial, sin descuidar el marco amplio en el que se conciben las ideas centrales para un cambio espacial duradero. 
En todo proceso de ordenación territorial, el desarrollo es la búsqueda de un escenario nuevo que resulte de la integración de estadios anteriores, los cuales, a su vez, evolucionan por separado. Es la idea de epigénesis, como la entiende Samaja (2009:136-137) valiéndose de un término que proviene de la embriología y que alude al estadio resultante de la evolución de partes en un todo integrado de partes que van creciendo cada una en su propio contexto. Así visto, el planeamiento territorial es una construcción de niveles a los que se suman procesos de acción y reacción para producir un cambio integral, de la misma manera que los órganos de un ser vivo van creciendo en la medida en que crece el conjunto.

En este proceso en que se suman las evoluciones de las partes para responder a un plan integrado es fundamental el papel de la geografía aplicada: cada nivel remite a una escala definida, independiente, pero a la vez genéticamente integrada. Esta forma holística de interpretar el desarrollo es propia de los geógrafos: los datos propios del crecimiento de cada espacio no son ajenos a la totalidad. La resultante esperada por el planificador es un paisaje complejo, una estructura territorial en la que convergen estadios espaciales conformados por sucesivos cambios impuestos por el hombre y sus respectivas respuestas unificadas en un todo.

En el plazo corto, son muy valiosas las experiencias realizadas a favor de la salud y el trabajo, porque las respuestas a los cambios se producen velozmente, lo que permite readecuar las estrategias en función de un modelo integral deseado, de plazo largo, producto de una dinámica epigenética.

Planificar para crecer es inherente a la idiosincrasia de los pueblos. «Dejar de crecer» es una opción prácticamente imposible. La apreciación subjetiva de los hombres es contraria a esta forma de pesimismo. El desarrollo, como forma de crecimiento, es una meta constante para las sociedades. Interrumpirlo es un acto - o, mejor dicho, lo contrario, la omisión de un acto- cercano a la idea nihilista de «dejar de vivir»... y perder la vida es perder la salud, perder el ambiente y perder el trabajo. Es por esta razón que se impone el refuerzo de sus variables componentes en los actos organizados en favor de la planificación, lo que es, en última instancia, una apuesta en favor de la vida. 


\section{Referencias bibliográficas}

Argentina (2008). Plan Estratégico Territorial. Primer avance, Buenos Aires: Alloni. (2010). Plan Estratégico Territorial. Bicentenario -I8I6, 2010, 2016-. Estado de Situación, Buenos Aires: San Carlos.

(20II). Plan Estratégico Territorial. Segundo avance, Buenos Aires: Letra Viva.

Braudel, F. (1958). «La longue durée», Annales, Economies, Sociétés, Civilisations, IV, 725-753.

Kossoy, M. (20I0). «Plan Estratégico Territorial 20I6», [en línea] recuperado de http://cdi.mecon.gov.ar/bases/ docelec/fdı26r.pdf

Labasse, J. (1973). La organización del espacio. Elementos de geografía aplicada, Madrid: Instituto de Estudios de Administración Local.

Pasquier, A. (1970). Où va la región?, Saint Armand: Dunod Actualité.

Popper, K. (1984). La miseria del historicismo, Madrid: Alianza-Taurus.

Randle, P. (1978). El método de la geografía, Buenos Aires: Oikos.

Rey Balmaceda, R. (1972). Geografía regional, teoría y aplicación, Buenos Aires: Estrada.

Roccatagliata, J. A. (1982). "El espacio desde la óptica geográfica», Aportes al pensamiento geográfico, I, 27-38.

Samaja, J. (2009). Epistemología de la salud. Reproducción social, subjetividad y transdisciplina, Buenos Aires: Lugar Editorial.

Sartre, J.-P. (2007). El existencialismo es un humanismo. Barcelona: Edhasa.

Soares de Olivera, A. M. (2007). «A urdidura do capital do trabalho nas áreas de Cerrado», Geografia e Trabalho no Século XXI, III, 86-87.

Weber, A. (1922). Über den Standort der Industrien.

Tübingen: Mohr-Paul Siebeck. 